\title{
La experiencia del caso de CEGRA: una propuesta de articulación en redes interuniversitarias e interinstitucionales
}

The experience of the CEGRA case: a proposal for articulation in inter-university and inter-institutional networks

\author{
Celia Cristina Basconzuelo ${ }^{1}$ \\ Universidad Nacional de Río Cuarto, Córdoba, Argentina
}

Recibido el 27 de junio de 2017, aceptado el 20 de julio de 2017

Disponible en internet el 12 de agosto de 2017

\section{Introducción}

En la República Argentina, la idea que explicita el compromiso social de la universidad pública reconoce su antecedente en los postulados básicos de la Reforma Universitaria, episodio emblemático de la Córdoba de los claustros. ${ }^{2}$ Los jóvenes que impulsaron tal movimiento estudiantil tenían claro que la docencia y la investigación debían estar acompañadas por las actividades de extensión, entendida por entonces como "proyección" hacia el medio.

En un pensamiento similar otros catedráticos se expresaron, inclusive desde el exterior. Así, el filósofo y ensayista español José Ortega y Gasset, en su obra Misión de la Universidad que publicó en 1930, reiteraba el principio de la pertinencia social de la educación superior. Tres décadas después, cuando el desarrollismo se tornó un paradigma económico alternativo para los países subdesarrollados, el sociólogo José Medina Echavarría sostenía que la universidad debía comprometerse con el desarrollo económico de su tiempo y con las demandas externas a sus muros. Más tarde, frente a los avances del neoliberalismo en América latina, la UNESCO se pronunció a favor del principio de la educación superior como bien público y destinado a desarrollar un papel relevante en los procesos de internacionalización. (Beltrán Llevador, 2014)

Esa tendencia continúa hasta hoy. En el actual siglo XXI, la universidad continúa atravesada por una tensión que la sitúa por una parte frente a las demandas sociales y por la otra frente a las presiones de la mercantilización del conocimiento y la privatización de los servicios educativos. Mientras los universitarios dirimen su posicionamiento como poseedores de una supuesta legitimidad del saber científico, continúan formándose profesionales, desarrollándose la investigación e impulsándose la extensión a un ritmo que no es homogéneo al comparar universidades de países

\footnotetext{
${ }^{1}$ Dra. en Historia. Investigadora Adjunta del CONICET. Correo electrónico: cegraunrc@gmail.com ${ }^{2}$ En 1918, durante la presidencia del radical Hipólito Yrigoyen, el movimiento estudiantil lanza una proclama desde la Universidad Nacional de Córdoba que iniciaría una gran reforma en las casas de altos estudios de todo el país, inclusive con influencia en varias universidades latinoamericanas. Allí, se sentaron los principios de la autonomía universitaria, el cogobierno, la periodicidad de las cátedras y los concursos de oposición.
} 
centrales y de los periféricos, además de una relación con el Estado y con el campo empresarial también disímil si se comparan las políticas universitarias y las científicas de regímenes populistas y de los liberales en los últimos cincuenta años de historia latinoamericana.

Empero, los retos pueden transformarse en oportunidades. En tal sentido, se cuenta con sugerentes aportes teóricos y empíricos dando cuenta de innumerables programas de cooperación y de internacionalización que convocan a la universidad y sus sujetos miembros. Al efectuar un balance, algunos destacan los significativos logros alcanzados en la materia (Sebastián, 2004); mientras otros, en cambio, concluyen que todavía son insatisfactorios. (Pedreño Muñoz, 2009) Una segunda perspectiva sobre la cual trabaja otra cantidad importante de proyectos remite a la relación de las universidades latinoamericanas con el entorno social; asunto respecto del cual también pueden adelantare resultados concretos y positivos.

Algunos autores destacan ese aporte de la universidad con la construcción social. (Malagón Plata, 2006) Otros focalizan en la cuestión del desarrollo, en tal sentido y como lo expresan Silvia Arias y Estefanía Molina la misión de la universidad "no sería colaborar sino intervenir, participar con implicación y hasta problematizar las situaciones dadas", con actitudes de "complementariedad y solidaridad". El fundamento es simple: el desarrollo es un esfuerzo compartido, cuya responsabilidad ya no debiera competir de manera exclusiva al Estado sino que además convocaría al resto de los actores sociales donde la Universidad está llamada a ser parte del mismo, "formando el capital humano, desarrollando el conocimiento y mejorando las condiciones en las que se despliega la dinámica social". (Arias y Molina, 2006: 18 y 31) Por su parte, Graciela Tonon continúa abonando esta perspectiva de la Universidad ligada al desarrollo de la comunidad, inclusive propugna su responsabilidad en la construcción de ciudadanía. (Tonon, 2012)

Para otros analistas resulta de mayor peso fundar la misión universitaria en el concepto "pertinencia social", de manera tal que le permita estar atenta "a los objetivos, necesidades y carencias de la sociedad en que está inserta y a las particularidades del nuevo contexto mundial". (Tünnermann Bernheim, 2001: 241) Según este autor, la universidad tiene una "misión cultural" y por ello debería contribuir a comprender y preservar las culturas nacionales y regionales, además de reforzar su función crítica y prospectiva mediante un análisis constante de las nuevas tendencias que atraviesan la sociedad, mediante un planteamiento interdisciplinario y transdisciplinario.

Así, pues, hay coincidencias entre los estudiosos del tema acerca de alentar y fundamentar una mayor participación de la educación superior en la sociedad contemporánea -sociedad del conocimiento y de la información- lo cual implica actualizar los objetivos, la misión y las funciones de las casas de altos estudios. Dentro de este contexto también se resignifica la extensión universitaria.

En la línea de innovaciones destacan las redes universitarias cuyo perfil y dinámica contrastan con la tradicional mirada de entender las relaciones interuniversitarias 
básicamente desde una óptica bilateral. La multilateralidad avanza como criterio reformativo en la medida que postula como criterios de base la cooperación. (Galarza López)

Este modo de concebir la extensión registra múltiples antecedentes en el mundo europeo; mientras, en el ámbito del Mercosur es reciente, aunque se han cosechado importantes logros. Particularmente, ello ha acontecido en el último quinquenio. ${ }^{3} \mathrm{Al}$ respecto, los relatos de las experiencias de integración regional son numerosos, dando cuenta de la importancia que adquiere la universidad como agente y actor de la cooperación. ${ }^{4}$

Por nuestra parte, el concepto "red académica" se discutió en un trabajo precedente. Se trataría de un mecanismo generador de flujos en el marco de una cultura de la interacción cooperativa entre universidades y con organizaciones e instituciones de la sociedad. Su propósito es la gestión en común del conocimiento científico en procura de un impacto social, toda vez que las sociedades contemporáneas, en virtud de su dinámica compleja, demandan respuestas más atentas a la diversidad, la multiculturalidad y la heterogeneidad. Respecto de su instrumentación, las redes permanecen en algunos casos en un estadio informal para luego confluir en la institucionalidad. En este caso, las opciones son diversas. Puede asumir la modalidad de una red investigativa interdisciplinar, de manera entonces que la investigación se constituye en el eje impulsor aunque se apoya al mismo tiempo en la función formativa docente y en la vinculación con el medio; o bien, integrar otras instituciones y organizaciones de la sociedad civil dando cuerpo a una red transversal universitariasocial, donde el eje es la extensión para nutrir la investigación y la docencia. (Basconzuelo y Souza Coelho, 2016)

Hacia ambas direcciones se alentó, en una universidad pública argentina, (Universidad Nacional de Río Cuarto) la creación del Centro de Estudios y de Gestión en Redes Académicas (CEGRA), constituyéndose en una estructura institucional que desde diciembre de 2016 pretende contribuir en la gestión de redes desde un rol articulador Universidad-Sociedad.

En virtud del contex to descrito, este artículo analiza aspectos teóricos y metodológicos que sustentan el estudio de caso. Así, el trabajo se encuentra estructurado en tres partes que abarcan dichos aspectos, donde la primera de ellas analiza el paradigma de la responsabilidad social universitaria (RSU), una matriz teórica cuyos postulados inspira la visión sobre la extensión universitaria que desarrolla nuestro Centro. La segunda, también de característica conceptual está basada en la noción "vinculación

\footnotetext{
${ }^{3}$ Los cuatro programas que integraron el sector educativo del MERCOSUR (PASEM, PPCP, POSGRADOS, NEIES) desarrollados entre 2011 y 2015 fueron un ejemplo de las políticas en tal sentido.

${ }^{4}$ Un registro de los mismos puede hallarse en los seis volúmenes de la Revista del Núcleo de Estudios e Investigaciones en Educación Superior del Mercosur, Integración y Conocimiento, ed itada desde 2011 a 2017.

Disponible en https://revistas.unc.edu.ar/index.php/integracionyconocimiento/issue/view/476/showToc
} 
con el medio" en virtud de la cual se explican, en la última parte del trabajo, las acciones emprendidas y se evalúan los resultados alcanzados hasta el presente.

\title{
ASPECTOS CENTRALES DEL PARADIGMA DE LA RESPONSABILIDAD SOCIAL UNIVERSITARIA (RSU)
}

El concepto "responsabilidad social" nació en Estados Unidos a finales de los años 1950 y principios de 1960 por referencia a las empresas. Por entonces, la expresión "responsabilidad social empresaria" pasó a designar una tendencia contrapuesta al modelo económico clásico, según el cual la única responsabilidad social de las entidades del sector era incrementar los beneficios de sus accionistas. Se postulaba, en contrapartida, un nuevo modelo de gestión donde las organizaciones empresarias no solo tuviesen como objetivo la maximización de los beneficios, sino además una disposición ética que guiase metas compatibles con el desarrollo sustentable de la sociedad y la preservación de recursos ambientales y culturales para las generaciones futuras. (Martínez y Soza, 2012)

A la par que el concepto iba cobrando fuerza teórica y empírica se expandió para alcanzar otras distintas organizaciones e instituciones y con el tiempo resultó aplicable también al quehacer de las universidades. Un hito histórico que abrió las puertas para una discusión sobre la responsabilidad social universitaria fue el pronunciamiento de la UNESCO, en julio del año 2009, a través de un documento redactado en el marco de la Conferencia Mundial sobre educación superior celebrada en París. Allí se expresó, entre otros principios, que

\begin{abstract}
la educación superior, en tanto bien público, es responsabilidad de todas las partes interesadas, en particular de los gobiernos [...] Los centros de educación superior, deberían centrarse aún más en los aspectos interdisciplinarios y promover el pensamiento crítico y la ciudadanía activa, contribuyendo así al desarrollo sostenible [...]La prestación transfronteriza de enseñanza superior puede representar una importante contribución a la educación superior. (UNESCO, 2009)
\end{abstract}

En los años posteriores a dicho evento fueron cobrando fuerza las argumentaciones que ensalzaban ese ideal de la participación universitaria sintetizada en la RSU. Así, el catedrático español Ricardo Gaete Quezada, señaló que dicha tendencia implicaba

\footnotetext{
desarrollar procesos de toma de decisiones y ejecución de acciones más conectadas con los intereses de la sociedad, asumiendo una actitud y un comportamiento permanente de su quehacer orientado hacia una filosofía organizacional, que asumía en su gestión los intereses propios, y a la vez los del entorno social (Gaete Quezada, 2001 ${ }^{\mathrm{a}}$ : 13; 2011b: 12).
}

Otros españoles se expresaron en una similar postura. Marta de la Cuesta González y David Sánchez Paunero -economistas de profesión- argumentaron con solidez acerca del compromiso universitario con la sociedad y a la vez con el desarrollo ambiental sostenible. Al respecto, sostenían

la RSU es un compromiso transversal, plural y multidimensional, inherente a la propia esencia de la actividad universitaria, no es algo meramente añadido a modo de filantropía. 
Fortalece la sociedad civil, dando mayor calidad y excelencia a una institución que es instrumento esencial para la cohesión social, la igualdad de oportunidades, la innovación y la investigación tecnológica y científica que son la base de la competitividad en la nueva sociedad del conocimiento, pero, además, para la construcción de los valores humanistas sobre los que hoy debe de asentarse nuestra sociedad (De la Cuesta y Sánchez, 2012: 10).

Según el filósofo francés François Vallaeys, la RSU comportaría "una nueva política de gestión universitaria". El rol socialmente responsable de la institución debiera manifestarse en la gestión de dos ejes. Uno organizacional, a partir del cual se percibiera que su instalación en el medio tiene un impacto laboral y medioambiental, lo que justificaría trabajar en pos de un campus ambientalmente sostenible. El otro eje debiera ser académico, de modo que la formación y la investigación se conecten con el entorno social y se emprenda una periódica revisión curricular a la luz de los desafíos socioeconómicos y ambientales contemporáneos. (Vallaeys, 2014)

En Latinoamérica, la venezolana Universidad de Zulia ha sido un referente en los últimos años de publicaciones que analizan la RSU. Una de sus investigadores, Cynthia Martínez de Carrasquero subraya que la vinculación con el entorno debiera articular necesariamente las funciones de docencia, investigación y extensión, citando como prácticas los servicios de consultoría y asesorías, entre otros. (Martínez de Carrasquero, 2011)

En Argentina, una de las catedráticas referente es Silvia Vázquez quien define entre los objetivos de la RSU los de "fortalecer las redes para intercambiar experiencias e intentar formar parte de las soluciones [...] y brindar respuestas pragmáticas a corto y mediano plazo para mejorar la sociedad”. (Vázquez, 2011: 2)

En síntesis, el paradigma propuesto por la RSU ha recogido numerosas teorizaciones, todas confluyentes en el aspecto de resaltar la necesaria vinculación de la Universidad con el medio social, su compromiso con prácticas que refuercen la sustentabilidad del desarrollo y principios de la ética aplicada. Se aleja bastante, por lo tanto, de una concepción meramente mercantilista o empresarial aunque también de aquellas visiones iniciales de la extensión universitaria entendida como "proyección". Posiciona a la universidad en un proceso dialógico de la construcción del conocimiento e interpela a quienes desde la sociedad y el Estado deseen comprometerse con la sociedad del conocimiento.

Ahora bien, este conjunto de axiomas puede permanecer en un hipotético plano de no mediar su plasmación en un proyecto que permita desenvolver en la práctica de articulación Universidad-Sociedad al menos algunos de sus principios. Para ilustrar cómo se piensa y concibe estas afirmaciones, se propone como estudio de caso la gestión del conocimiento instrumentada desde el CEGRA. 


\section{REVISITANDO LA NOCIÓN DE EXTENSIÓN UNIVERSITARIA: HACIA LA "VINCULACIÓN CON EL MEDIO"}

¿Qué tipo de compromisos puede asumir la universidad a partir de poseer un "capital intelectual acumulado". ${ }^{5}$ La pregunta, con base en un concepto bordeuniano, interpela a la comunidad de universitarios y abre un abanico de posibles respuestas desde las clásicas, donde quedan comprendidas la docencia y la investigación básica y aplicada, hasta los planos prospectivos donde resalta aún más el plano aplicativo de la ciencia y las áreas estratégicas.

Pensar la extensión desde una perspectiva renovada, significa trasponer aquella visión mediante la cual los académicos entendían que la misión de los claustros era "proyectar" sus actividades y "comunicar" los resultados de sus investigaciones, para modificar cualitativamente la mirada $\mathrm{y}$, por consiguiente, proponer el término "vinculación con el medio".

Tal apreciación se nutre de algunos supuestos básicos que se enuncian aquí a modo de hipótesis sobre la base de debates enriquecidos a partir de experiencias de intercambio y discusiones en eventos académicos recientes:

a- La universidad es un actor institucional componente de un entramado social que contextualiza su desenvolvimiento pasado, enmarca su presente y la interpela hacia su futuro. Sostenemos esta idea pues el conocimiento científico no debiera construirse solamente "desde la universidad", sino además desde la sociedad, junto con esos otros actores $u$ organizaciones a quienes pueden resultar de interés las problemáticas de investigación y de vinculación, así como los contenidos que se imparten en la formación profesional que brindan los centros de estudios superiores. Este supuesto encuentra en el mecanismo de las redes del conocimiento ${ }^{6}$ una de las expresiones más concretas, reservándose la expresión redes académicas para ejemplificar la cooperación nacional e internacional sobre las bases interuniversitarias. La expresión antedicha no pretende subalternizar la construcción de saberes sólo a la matriz académica, sino que invita al diálogo con todos los actores que puedan favorecer la producción de un conocimiento válido. ${ }^{7}$

b-Vinculación con el medio no es sinónimo de "transferencia" de las producciones académicas, tal como tradicionalmente se entendía la extensión. La interacción con el medio implica otros desafíos. No se trata de reaccionar sino de influir mediante acciones vinculantes que refuerzan la autonomía universitaria y que comprometen. En palabras del catedrático argentino Carlos Coppa, ese compromiso lo es

\footnotetext{
${ }^{5}$ La expresión es de Bordieu, Pierre. (2000). Los usos sociales de la ciencia. Buenos Aires: Nueva Visión Argentina.

${ }^{6}$ Obsérvese la utilización de la expresión redes del conocimiento y no solo "redes académicas" a los fines de no sujetar la vinculación con el medio a una matriz subalterna de lo académico.

${ }^{7}$ Esta premisa es formulada teniendo en cuenta, por ejemplo, el anclaje de algunas universidades en proximidad con comunidades aborígenes, con las cuales puede dialogar para el diseño de políticas culturales receptoras y más inclusivas de esos otros saberes comunitarios.
} 
con el desarrollo del pensamiento crítico, la opción por producir y difundir conocimientos socialmente válidos, y el involucramiento directo en acciones o programas que incidan en problemáticas específicas del entorno sociopolítico y cultural. (Coppa, 2013: 8)

c- La vinculación con el medio se fundamenta en un principio que valoriza lo relacional porque se entiende que lo complejo es un rasgo constitutivo de la realidad social, de manera que una perspectiva coherente con ello debería observar una necesaria convergencia de saberes científicos, de enfoques y metodologías interdisciplinares. Esta premisa implica que en las redes académicas se asocian y dialogan las ciencias sociales, aunque pueden -y deberían- intervenir todos los campos científicos. La amplitud de miradas se deriva de la característica de complejidad que en la actualidad se atribuye a lo social.

El paradigma de la complejidad es autoría del filósofo Edgar Morín quien a finales del siglo XX apeló a la teoría de los sistemas y de la información para enunciar las principales líneas que dieron sustento a un enfoque desde el cual muchos pretenden orientar el abordaje de la ciencia contemporánea. Según sus ejes centrales, lo complejo sería un atributo de la realidad, no existiría una separación absoluta entre el sujeto y el objeto del conocimiento, los valores también integrarían la cognición humana y la responsabilidad se constituiría en un elemento de la producción de conocimientos científicos. (Taeli, 2012)

La validez de los estudios unidisciplinares comenzó a ser puesta en entredicho por su eficacia para explicar lo complejo; de allí que se insistiera en la pertinencia de las metodologías holísticas y por eso, el énfasis en lo interdisciplinar. Derivación que permitió redescubrir a Jean Piaget, quien en los años 1970 ya había anticipado respecto de tres niveles crecientes de interrelación entre las ciencias. Uno inferior, multidisciplinar, cuando en la solución de un problema aportan su análisis varias disciplinas, sin que ello derive en cambios teóricos o de modelos para las ciencias involucradas; luego, un segundo nivel donde se registra la cooperación entre varias disciplinas y que conduce a interacciones mutuas de conceptos y de métodos. Finalmente, en un tercer nivel, la transdisciplinareidad, cuando se consigue desdibujar las fronteras entre las disciplinas. (citado por Tünnermann, 2001: 112)

\section{Como sostiene Adela Cortina}

es el trabajo interdisciplinar el que mostrará de nuevo el hilo conductor de la unidad del saber y el que ayudará a resolver los problemas que desbordan las posibilidades de cada ámbito epistemológico determinado (Cortina, 2005: 19).

Transitar hacia un estadio transdisciplinar supone alcanzar un conocimiento superior, fruto de un movimiento dialéctico de retro- y pro-alimentación del pensamiento, después de atravesar las fronteras de diferentes áreas del conocimiento disciplinar, lo que encamina un diagnóstico de la realidad más completo e integrado. (Martínez Migueles, 2007) 


\section{ESTUDIO DE CASO: APORTES DESDE UN CENTRO DE ESTUDIO Y DE GESTIÓN EN REDES ACADÉMICAS}

En la convicción que la universidad pública debe participar activamente en el desarrollo económico, social y cultural territorial y asumir una responsabilidad cada vez mayor en materia de formación, investigación, estudio y servicios de transferencia, es que se creó un Centro de Gestión de Redes en el ámbito de la Universidad Nacional de Río Cuarto, por iniciativa de investigadores de las ciencias sociales, de carácter ciertamente innovador en el área. ${ }^{8}$

A poco de iniciar su gestión, fueron instrumentándose algunas bases de ese paradigma de la RSU. La primera fue diseñar Nodos de Investigación que respondiesen a varias áreas estratégicas de interés social, más acorde ya no solo con los lineamientos estratégicos a nivel nacional, de la cual por cierto esa universidad pública y estatal, es parte, sino básicamente con su entorno territorial más próximo donde se halla localizada. Es decir, que el criterio que se observó al integrar esos Núcleos fue la conformación multidisciplinar para abordar problemáticas en un nivel interdisciplinar y aspirar a logros transdisciplinares.

Así, los Núcleos abordarían los estudios de la economía social, género y desarrollo sustentable, la acción colectiva, la participación ciudadana y las políticas públicas, los conflictos socio-ambientales, el medio ambiente y las redes de conocimiento. Estos campos de investigación social se concibieron integrados con la formación profesional, pues el equipo de investigadores reúne personal capacitado en la universidad con nivel de posgrados (Maestrías y Doctorados) así como en el CONICET (investigadores de carrera y becarios).

Un segundo paso que se dio en el CEGRA fue gestionar proyectos en redes, acción que aún continúa y se constituye en la dinámica de base pues hace visible el compromiso social de la universidad pública. (Ver Diagrama de Redes)

Por un lado, esas redes comprenden a las universidades nacionales y extranjeras que se asocian cooperativamente a los Núcleos de Investigación, junto con Centros de Estudios o de Investigación del país o del exterior. El objetivo que las dinamiza es, por una parte, la realización de proyectos de investigación asociados a una temática convocante de perspectivas interdisciplinares. Por otra parte, se busca cubrir la demanda de actualización académica y formación en posgrado mediante convenios de cooperación, permitiendo así avanzar en áreas de fortalecimiento recíproco y complementariedad, titulaciones de doble reconocimiento, además de las acciones más tradicionales como la realización de eventos científicos conjuntos y la movilidad docente-estudiantil.

\footnotetext{
${ }^{8}$ De los 11 Centros de Investigaciones y Transferencia que pertenecen al CONICET, 4 representan aunque no exclusivamente el campo de aplicación social. Véase http://red.conicet.gov.ar/listado-cit/
} 
Por otro lado, las redes se construyen trazando vínculos con instituciones del medio: Municipalidad ${ }^{9}$, organizaciones sociales, instituciones educativas. ${ }^{10}$ De ese modo, la universidad visibiliza la voluntad de constituirse en actor estratégico en la agenda del desarrollo local desde una posición dialógica, ya que los investigadores responsables de los Nodos interrelacionan con los actores socio institucionales y conocen cuáles son las demandas y problemáticas que merecen convertirse en "sujetos de estudio". Las herramientas que permiten viabilizar esas redes son los convenios marcos y los protocolos específicos en los cuales se explicitan y detallan las acciones a seguir.

El CEGRA transita actualmente una etapa experimental. Los logros evidentes se dan en el campo de conformación de los núcleos de investigación y en los proyectos de vinculación con el medio diagramados. Resta por delante un camino de compromiso con esta línea de responsabilidad universitaria por el conocimiento y de mayor articulación con actores de la sociedad local.

\section{A MODO DE CONCLUSIÓN}

A modo de cierre, puede expresarse que si la universidad es considerada en su doble papel de actor institucional y social, no puede tener una presencia menos relevante en el medio local y regional. Ello demandaría algunos desafíos. El primero, es de carácter posicional: es preciso salir de la comodidad académica, ir a la búsqueda de los problemas sociales en una actitud dialógica con otros actores sociales y con otras instituciones, ensayar ideas prospectivas y no solo hipótesis que puedan acaso ser apropiadas por quienes diseñan políticas públicas en el territorio. Segundo, construir redes académicas y del conocimiento es articular saberes, y no solo redes. El conjunto de las ciencias y las sociales en particular cuentan con arsenales conceptuales y teóricos válidos para participar en una construcción cooperativa del conocimiento, edificadora de un habitus intelectual sobre la base del diálogo interdisciplinar, la indagación multidisciplinar y el horizonte transdisciplinar.

Articular, vincular, dialogar, ¿para qué? En esta búsqueda del sentido puede recordarse una antigua reflexión weberiana condensada en la distinción entre ciencia y política. Ambas son vocacionales, diría Weber (2012), y el sentido radica en entenderlas como "servicio", "compromiso constante" y "responsabilidad". Como predica también Boaventura de Souza Santos (2007) la ciencia debería aproximarse a la ética y los problemas tal vez no son solamente teóricos sino epistemológicos.

Podría añadirse que en la sociedad del conocimiento, es la hora del protagonismo de los cientistas sociales con su arsenal de teorías y epistemologías, las cuales tal vez

\footnotetext{
9 Con el Municipio se ha avanzado en dos proyectos de capacitación: en un caso destinado a funcionarios públicos sobre áreas de interés para los mismos que fueron delimitadas tras sucesivas reuniones de diálogo; en el otro, destinado a operadores turísticos y centrado en Prácticas de Turismo Sustentable.

${ }^{10}$ Con este tipo de instituciones se han diagramado cursos destinados a educadores del nivel medio y superior de la ciudad.
} 
deban ser revisitadas y reactualizadas a la luz de las realidades y problemáticas contemporáneas.

Con seguridad, la universidad resultará beneficiada. Una oportuna y periódica actualización de los planes de estudio, propuestas de investigación aplicada en ciencias sociales, trayectos curriculares novedosos, definición de áreas estratégicas locales, pueden resultar de un proceso necesario y a la vez virtuoso de retroalimentación Universidad-Sociedad. Sortear esos desafíos significaría estar en el camino del paradigma de la RSU. Plantearlos, al menos, implicaría considerarlo como un punto cardinal posible para la educación superior del siglo XXI.

\section{Diagrama de Redes (CEGRA)}

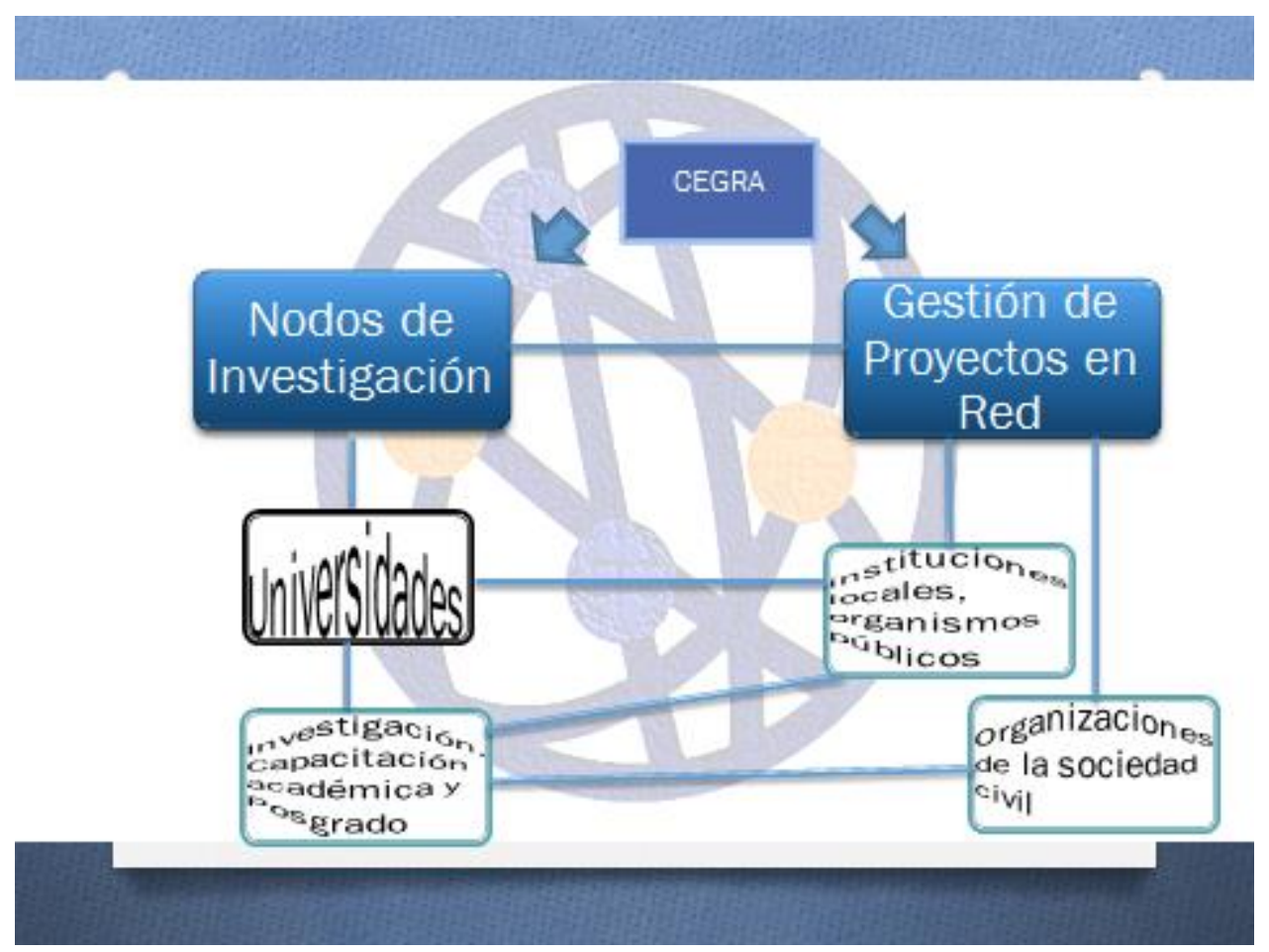

\section{Bibliografía consultada}

Arias, Silvia y Estefanía Molina (coords). (2006). Universidad y cooperación al desarrollo. Cuadernos solidarios $N^{\circ} 1$. Madrid: UAM.

Basconzuelo. Celia y Agripino Souza Coelho Neto. (2016). Redes Académicas Interuniversitarias en Mercosur: el papel de la extensión. En Integración y Conocimiento. $\mathrm{N}^{\circ}$ 5. Vol. 2. Buenos Aires: NEIES, pp. 98-110

Beltrán Llevador, José. (2014). La responsabilidad social universitaria, el reto de su construcción permanente. En Revista Iberoamericana de Educación Superior. Vol. 5, pp. 3-18.

Disponible en http://www.sciencedirect.com/science/article/pii/S2007287214702975 
Coppa, Carlos (2013). La vinculación con el medio social como categoría para la evaluación y acreditación universitaria. En Anuario de la Facultad de Ciencias Económicas del Rosario. № 9. Disponible en: http://bibliotecadigital.uca.edu.ar/repositorio/revistas/vinculacion-medio-socialcomo-categoria.pdf

Cortina, Adela. (2005). La misión de la universidad: educar para la ciudadanía del siglo XXI. Conferencia de inauguración del año académico. Pontificia Universidad de Valparaíso.

Disponible en http://archivohistorico.ucv.cl/files/discursos/Inauguracion_2005.pdf

De la Cuesta González y Sánchez Paunero, David. (2012). Responsabilidad social universitaria 2.0. La Coruña: Netbiblo

Galarza López, Gabriel. (2010). Las Redes Universitarias en el Desarrollo de la Educación Superior. En: Boletín IESALC Informa. $\mathrm{N}^{\circ} 210 . \quad$ Disponible en http://iesalc.unesco.org.ve/index.php?option $=$ com content $\&$ view $=$ article \&id $=2139 \% 3$ Alas redes-universitarias-en-el-desarrollo-de-la-educacion-superiorlas-redes-universitarias-en-eldesarrollo-de-la-educacion-superior\&catid=126\%3Anoticias-paginanueva\&Itemid=712\&lang=es

Gaete Quezada, Ricardo. (2011a). Responsabilidad social universitaria: una nueva mirada a la relación de la universidad con la sociedad desde la perspectiva de las partes interesadas. Un estudio de caso. Universidad de Valladolid. Tesis Doctoral.

Disponible en https://uvadoc.uva.es/bitstream/10324/923/1/TESIS148-120417.pdf

Gaete Quezada, Ricardo. (2011b). La responsabilidad social universitaria como desafío para la gestión estratégica de la educación superior: el caso de España. En Revista de Educación. $\mathrm{N}^{\circ}$ 355, pp. 109-133. Disponible en http://www.revistaeducacion.educacion.es/re355 05.html

Malagón Plata, Luis Alberto. (2006). La vinculación Universidad-Sociedad desde una perspectiva social. En Educación y Educadores. Volumen 9, Número 2, pp. 79-93. Colombia: Universidad de La Sabana. Disponible en www.redalyc.org/pdf/834/83490210.pdf

Martínez, Mónica y Carlos Soza. (2012). La responsabilidad social de las empresas: orígenes, aproximaciones conceptuales y estrategias.

Disponible en http://fhu.unse.edu.ar/carreras/rcifra/martinez_soza.pdf

Martínez de Carrasquero, Cynthia. (2011). Responsabilidad Social Universitaria y su Articulación con las Funciones Docencia-Investigación-Extensión para su Vinculación con el Entorno Social. En Boletín IESALC Informa. $\mathrm{N}^{\circ} 214$.

Disponible

en http://iesalc.unesco.org.ve/index.php?option=com content\&view=article\&id=2595\%3Aresponsa bilidad-social-universitaria-y-su-articulacion-con-las-funciones-docencia-investigacionextension-para-su-vinculacion-con-el-entorno-social\&catid=126\%3Anoticias-paginanueva\&Itemid $=712 \&$ lang $=$ es

Martínez Miguélez, Miguel. (2007). Conceptualización de la transdiciplinariedad. En Polis. Revista Latinoamericana. $\mathrm{N}^{\circ} 16$.

Disponible en http://polis.revues.org/4623

Pedreño Muñoz, Andrés. (2009). Internacionalización del conocimiento. El papel de las nuevas tecnologías y las universidades. En Utopías y realidades.

Disponible en http://utopias-realidades.blogspot.com.ar/2009/03/internacionalizacion-delconocimiento.html

Sebastián, Jesús. (2004). Cooperación e internacionalización de las universidades. Buenos Aires: Biblos. Siufi, Gabriela. (2010). La cooperación Internacional en la Educación Superior en Latinoamérica. En: Boletín IESALC Informa. $\mathrm{N}^{\circ} 211$

Souza Santos, Boaventura de (2007). Los desafíos de las ciencias sociales hoy. Disponible en http://bibliotecavirtual.clacso.org.ar/ar/libros/coedicion/boavent/cap\%203.pdf

Taeli Gómez Francisco. (2012). El nuevo paradigma de la complejidad y la educación: una mirada histórica. En Polis. Revista Latinoamericana. $\mathrm{N}^{\circ} 25$.

Disponible en http://polis.revues.org/400

Tonon, Graciela. (2012). Las relaciones universidad-comunidad: un espacio de reconfiguración de lo público. En Polis. Revista Latinoamericana. $\mathrm{N}^{\circ} 32$.

Disponible en http://polis.revues.org/6691 
Tünnermann Bernheim, Carlos. (2001). Universidad y sociedad. Balance histórico y perspectivas desde América Latina. Managua: Editorial Hispamer

UNESCO. (2009). Conferencia Mundial sobre educación superior.

Disponible en http://www.unesco.org/education/WCHE2009/comunicado_es.pdf

Vázquez, Silvia. (2011). Mitos y objetivos de la Responsabilidad Social Universitaria. En Boletín IESALC Informa. $\mathrm{N}^{\circ} 214$

Vallaeys, François (2014). La responsabilidad social universitaria: un nuevo modelo universitario contra la mercantilización. En Revista Iberoamericana de Educación Superior. Vol. 5, pp. 105-117.

Disponible en http://www.sciencedirect.com/science/article/pii/S2007287214719456

Weber, Max. (2012) [1920]. El político y el científico. Madrid: Alianza Editorial 